\title{
Management of Neonatal Teeth: Two Case Reports
}

\author{
Claudia Lobelli Chandler ${ }^{1}$, Manoelito Ferreira Silva Junior ${ }^{2 *}$, Maria da Conceição Pereira Pinto Solano ${ }^{3}$ and \\ Isabelita Duarte Azevedo ${ }^{4}$ \\ ${ }^{1}$ Doctor of Dental Sciences, University of Colorado School of Dental Medicine, USA \\ ${ }^{2}$ Collaborate Professor, State University of Ponta Grossa UEPG, Brazil
}

${ }^{3}$ Coordinator of the specialty training program of the Brazilian Dental Association, Natal, Rio Grande do Norte, Brazil.

${ }^{4}$ Associate Professor at the Federal University of Rio Grande do Norte UFRN, Natal, Rio Grande do Norte, Brazil

*Corresponding author: Manoelito Ferreira Silva Junior, Collaborate Professor, State University of Ponta Grossa UEPG, Brazil

Received: 粡 February 21, 2020

Published: 笽 March 06, 2020

\begin{abstract}
Neonatal teeth are abnormalities in the chronology of eruption in which one or more teeth, which erupt during the first 30 days following birth and cause problems such as trauma-induced ulcers of the tongue, damage to the mother's breasts, a reduction in the quality of breastfeeding, weight loss and even aspiration of the neonatal tooth. The removal or not of these teeth should be assessed on a case-by-case basis. The aim this article was to report the clinical management of two cases of neonatal teeth. The two female babies were attended in a pediatric dental clinic. The first patient was a 2-month-old baby that had difficulty during breastfeeding due to the mobility of one of the neonatal teeth. The clinical and radiographic exam showed that the three teeth were part of the normal dentition. The two mandibular teeth had approximately one third of the crown erupted and moderate mobility and opted for follow-up. The maxillary tooth was fully erupted and had severe mobility and we opted for its removal. The clinical and radiographic exam confirmed that the two teeth were part of the natural dentition. The teeth presented the clinical crowns fully erupted and both mobile, the left tooth having more mobility than that of the right tooth and opted for removal of more mobile left central incisor. In both cases, the extractions of teeth with high mobility were performed by greater risk of aspiration and caused more difficulty during breastfeeding. The follow-up showed a quick recovery, easier breastfeeding, including weight gain.
\end{abstract}

Keywords: Neonatal teeth; newborn; pediatric dentistry

\section{Introduction}

It is important that pediatricians and pediatric dentists are knowledgeable about the normal characteristics and anomalies in a newborn baby's oral cavity, as a foundation for early diagnosis $[1,2]$. One of the dental anomalies found in a newborn's oral cavity is natal and/or neonatal teeth, whose prevalence is one in every 3,000 live births [1]. Natal teeth are present at birth, while neonatal teeth appear in the oral cavity during the first 30 days of life [3]. The etiology of these teeth can range from hereditary factors, or a superficial position of the tooth germ, infections or even as a sequela of congenital syphilis [3-7]. There is evidence of a relationship between these anomalies and Pierre Robin [7] syndrome and also with premature and low birthweight children [8]. It is important to determine if the tooth is part of the normal dentition or if it is a supernumerary. If a radiograph confirms the tooth is a supernumerary, extraction is indicated, as well as in cases of excessive mobility, which can present a risk of aspiration [4-12]. If an extraction is indicated, it should be performed after the first week of life. Prior to this, the baby is incapable of absorbing vitamin $\mathrm{K}$ and the breast milk is lacking in this vitamin. To avoid bleeding, surgical procedures before this age should be preceded by vitamin $\mathrm{K}$ supplementation [6]. These teeth can also be associated with ulcers forming on the tongue surface in newborns, known as Riga-Fede $[3-5,13,14]$, which can lead to nutritional deficiencies in the baby $[2,5,11,15]$. Therefore, clinical management should be evaluated with discretion and on a case-by-case basis. In this sense, the aim this article was to report the clinical management of two cases of neonatal teeth.

\section{First Case Report}

A female patient, dark skinned, full-term birth, two months old, was attended to the pediatric dental clinic of the Brazilian Association of North Rio Grande. Mother's main complaint was the presence of three teeth that appeared when the child was less than one month old and the difficulty, they caused during 
breastfeeding due to the excessive mobility of one of the teeth. At the time of the consult, the child weighed less than expected for her age. According to the mother, the child was born with bumps on both the maxilla and mandible, in the area of the future central incisors. After 15 days, two lower teeth erupted, right first, then the left. After 20 days, an upper tooth erupted. The mother denied any family history of neonatal teeth. During the intraoral clinical exam, two mandibular incisors and one maxillary incisor were observed
(Figure 1A). The two mandibular teeth had approximately one third of the crown erupted and moderate mobility. The maxillary tooth was fully erupted and had severe mobility (Figures 2A \& 2B). No lingual ulcer was found during the exam. Two radiographs, upper and lower anterior regions, were taken using a \#2 film and a modified occlusal technique. The exposure time was reduced by one half ( 0.2 seconds) and the baby was placed in a supine position, in the mother's lap. Both used a lead apron for radiation shielding.

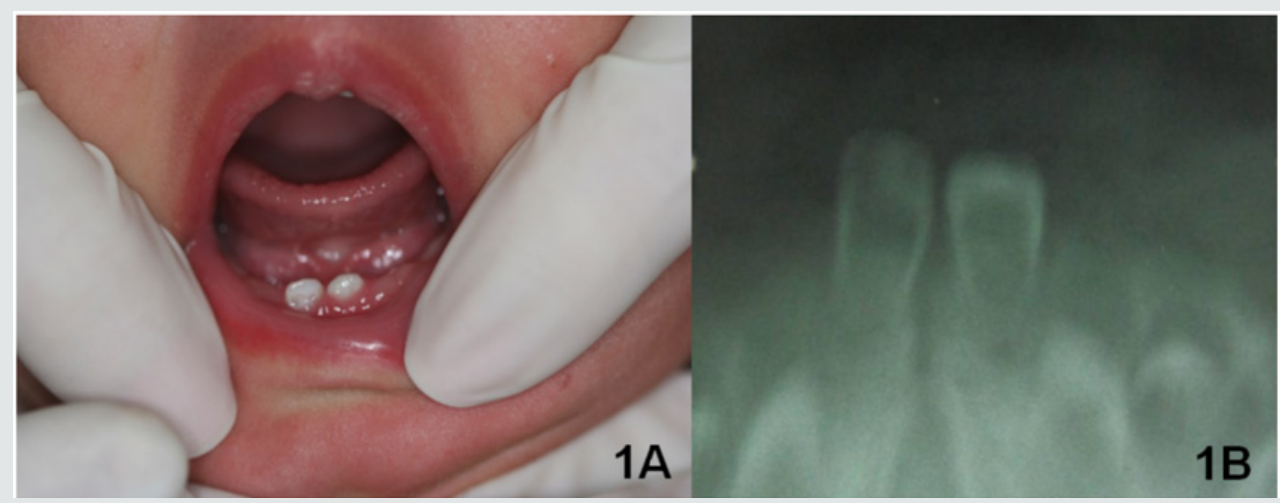

Figure 1: A) Neonatal teeth in the region of lower central incisors and lack of ulcer on the ventral surface of the tongue; B) Lower occlusal radiograph showing neonatal lower incisor teeth.

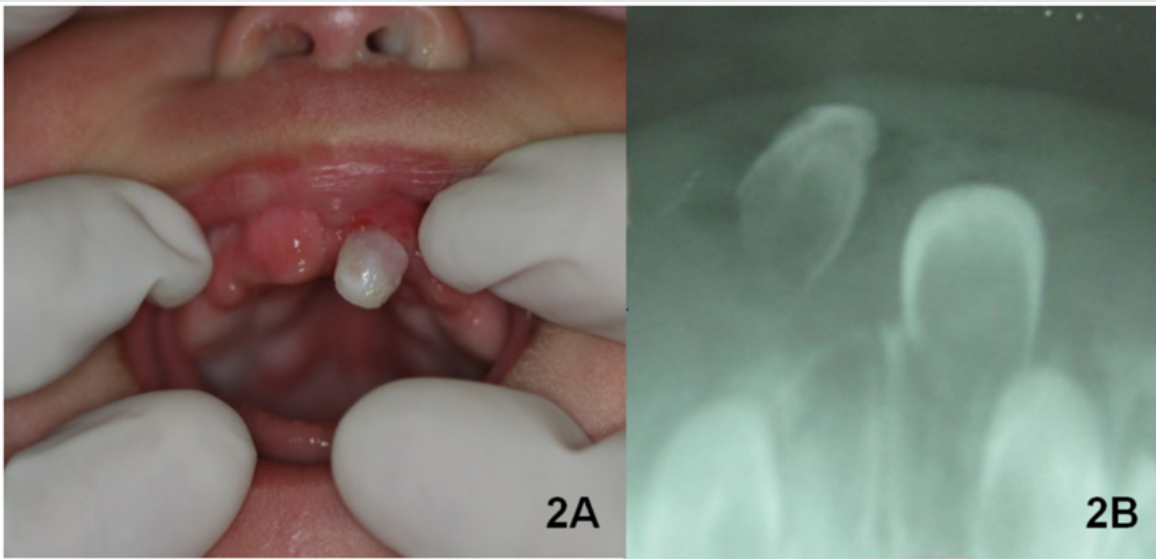

Figure 2: A) Neonatal tooth in the region of upper left central incisor and bump in the region of upper right central incisor; B) Upper occlusal radiograph showing the neonatal teeth in the region of upper left central incisor.

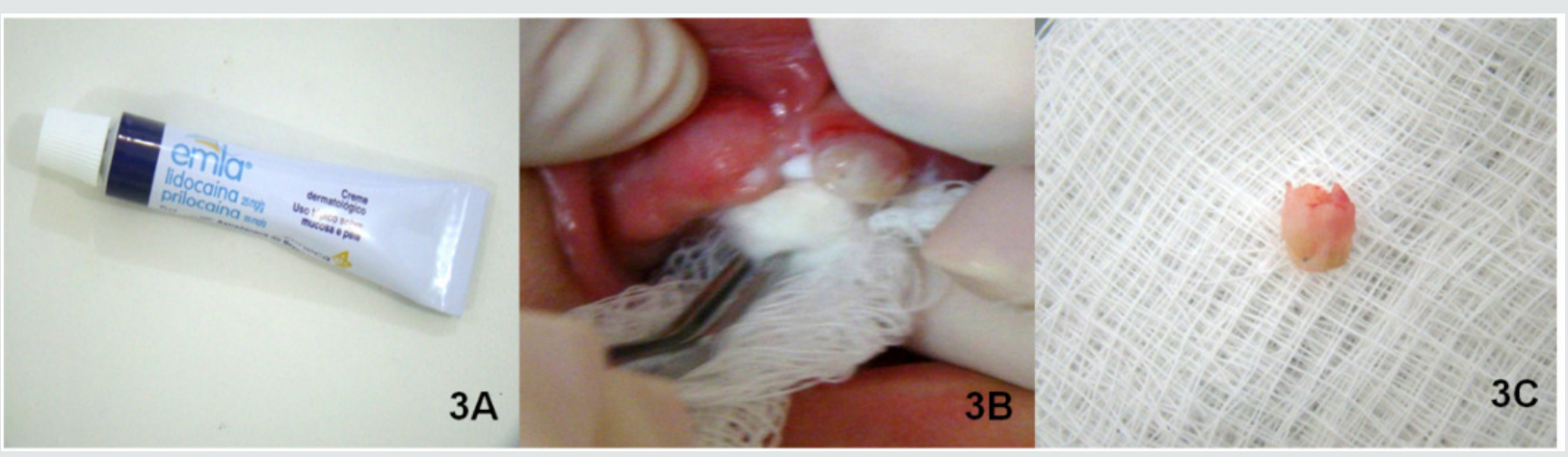

Figure 3: A) Topical anesthetic EMLA (Lidocaine Hydrochloride 2.5\%).

B) Topical anesthesia, being careful to avoid that the baby could swallow the anesthetic.

C) Neonatal tooth in the region of upper left central incisor extracted. 
The radiographs suggested that the deciduous teeth were part of the normal dentition. The decision was made to remove the maxillary tooth because, according to the mother, the child had difficulty latching on during breastfeeding due to the high degree of mobility of this tooth, resulting in both irritability and weight loss (Figures 1B \& 2B). The mother was informed about the recommendations for treatment, along with risks and benefits after which she gave written authorization to proceed with treatment, photographic documentation and scientific publishing. The procedure was performed with the dentist and mother in the knee-to-knee position, and the baby reclined in the dentist's lap.
No vitamin K prescription prophylaxis was performed because the baby had already achieved the normal safe levels. Initially, the mucosa was dried and a topical anesthetic (EMLA - lidocaine hydrochloride 5\%) (Figure 3A) was applied with cotton three times, being cautious to prevent its ingestion by the baby. The extraction was performed with sterile gauze (Figure 3B \& 3C). Cotton gauze was applied with pressure for hemostasis and the mother was told to immediately begin breastfeeding because the breast milk contains immunoglobulins which promote healing and the intimate contact with the mother provides security to the baby [4] (Figure $4 \mathrm{~A} \& 4 \mathrm{~B})$.

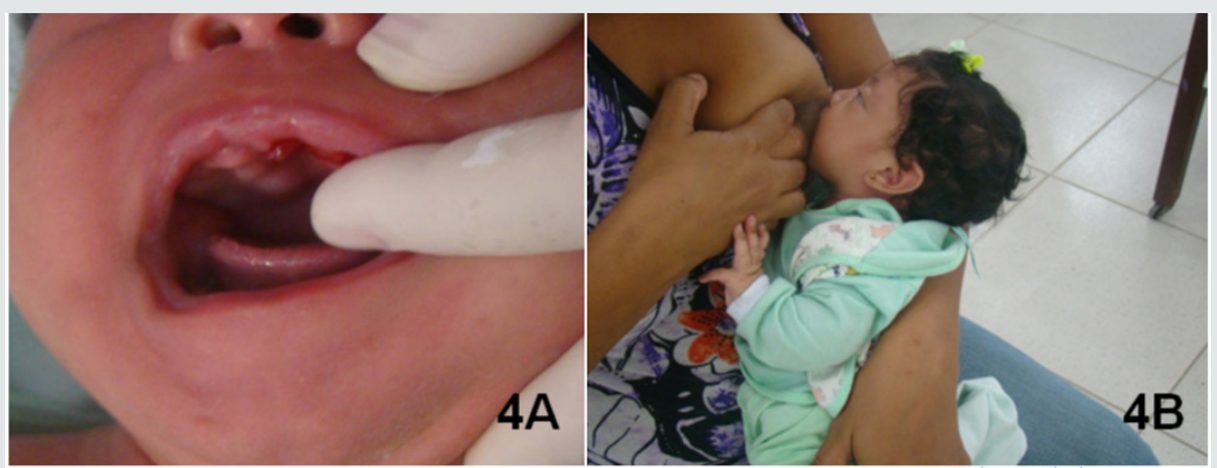

Figure 4: A) Clinical appearance immediately after the extraction.

B) Child being breastfed, just after the extraction. The breastmilk has a hemostatic effect and helps to calm the baby.

The day after the procedure, the mother was contacted by phone and she reported that there was a small clot in the area of the extraction. She also indicated that the child latched on more easily during breastfeeding and the absence of any irritability. The next day, the mother was again contacted by phone and she reported that the clot was gone. After seven days the healing was complete, and within thirty days the child had gained weight.

\section{Second Case Report}

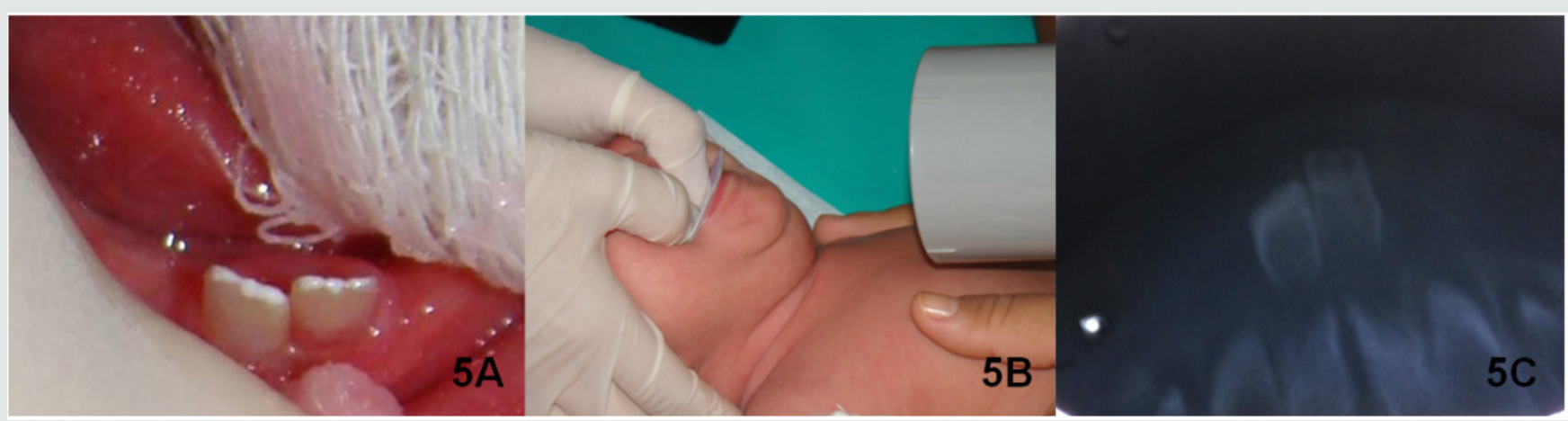

Figure 5: A) Neonatal teeth in the region of lower central incisors and lack of ulcer on the ventral surface of the tongue.

B) Taking the radiograph using the modified occlusal technique.

C) Radiograph of two neonatal teeth in the region of lower central incisors.

A 30-day-old white female patient was seen at the same dental center as the previous case. The guardian reported the presence of neonatal teeth as the primary concern, along with difficulty breastfeeding due to the mobility they presented. According to the mother's report, the child was born with a bump in the region of the lower incisors and two days later the bump disappeared, replaced by two teeth. These teeth initially presented with slight mobility, which gradually worsened over time. The patient had a family history of neonatal teeth with the aunt having the same condition when born. Mother also reported the child was gaining less weight than was expected at that age. Upon performing an intraoral clinical examination, two teeth were seen in the mandibular region corresponding to the area of the lower central incisors (Figure $5 \mathrm{~A})$. The teeth presented with the clinical crowns fully erupted and both mobile, the left tooth having more mobility than that of the right tooth. The soft tissue surrounding the teeth appeared normal and the tongue and sublingual region demonstrated no ulceration. Two radiographic films were taken using the same 
protocol as in the first described case (Figure 5B). The radiographs confirmed that the two teeth were part of the natural dentition (Figure 5C). The mother was informed of the recommendations for the treatment, along with risks and benefits after which she gave written authorization to proceed with treatment, photographic documentation and scientific publishing. The mother opted for removal of more mobile left central incisor, which had a greater risk of aspiration and caused more difficulty during breastfeeding. The extraction was performed following the exact same protocol as the first case (Figure 6). Additionally, the same healing process occurred as in the first case. We followed-up by phone the day following and one week following. Then one month after the exam, the patient returned to the clinic where the right mandibular central incisor presented with no increase in mobility (Figure 7).

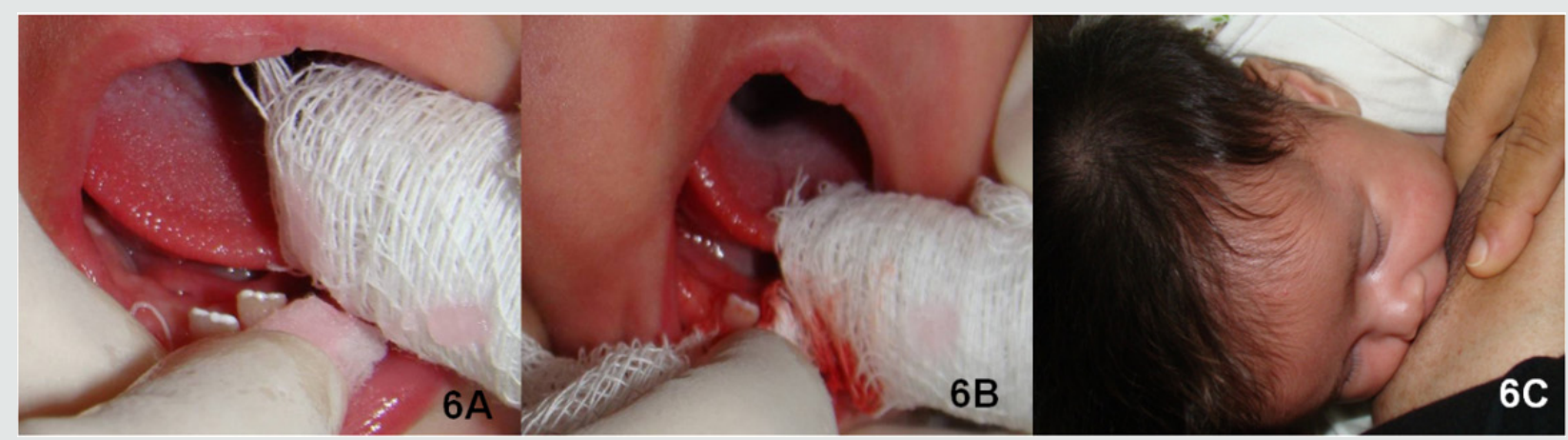

Figure 6: A: Topical anesthesia, being careful to avoid that the baby could swallow the anesthetic; B: Lower left central neonatal tooth being extracted. C: Child being breastfed, just after the extraction.

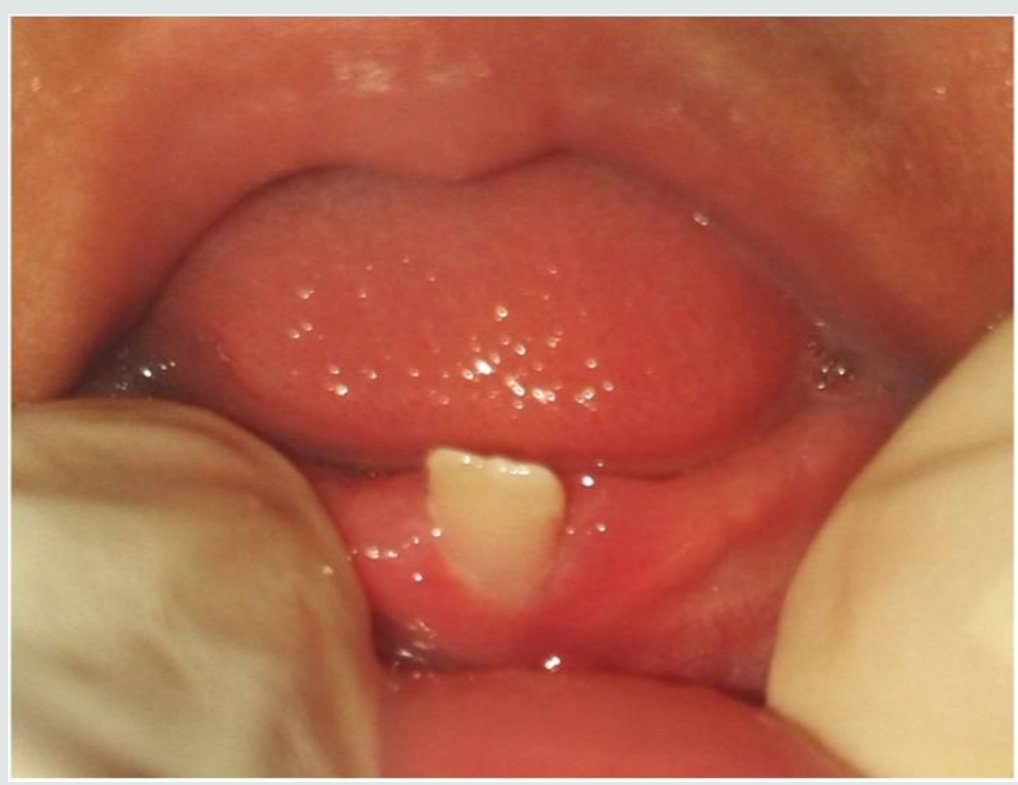

Figure 7: Healing one-month post-extraction.

\section{Discussion}

The included clinical cases reference the occurrence of upper and lower central incisor neonatal teeth. The epidemiological data shows that these eruption anomalies are most common in lower central incisors, around $85 \%$ of the cases, and upper central incisors in around $11 \%[6,7,16]$. According to Cunha et al. [15], natal and neonatal teeth are often seen in pairs, which is corroborated in the second case presented. The two patients were female, according to the literature this is the more affected sex $[3,6,7,16,17]$. The etiology of these teeth is still unknown [18]. Nevertheless, some authors report that it may or may not be a genetic condition $[6,12,17]$. The increased gain in body weight that was observed after the surgical extractions was similarly reported by other authors $[4,5,7,13,14,19]$. These cases and the benefits seen by both the mother and patient reiterate the importance of early management by a dentist. Neonatal teeth are often associated with excessive mobility and weight loss, affecting the baby's health and quality of life.

\section{References}

1. Abanto J, Raggio DP, Alves FBT, Corrêa FNP, Bönecker M, Corrêa MSN (2009) Oral characteristics of newborns: report of some oral anomalies and their treatment. Int J Dent 8(3): 140-145.

2. Diniz MB, Gondim JO, Pansani CA, Abreue Lima FCB (2008) A importância da interação entre odontopediatras e pediatras no manejo de dentes natais e neonatais. Rev Paul Pediatr 26(1): 64-69. 
3. Adekoya Sofowora CA (2008) Natal and neonatal teeth: a review. Niger Postgrad Med J 15(1): 38-41

4. Sabás M, Pascual DM, Frelos MPP (2001) Dientes natales y neonatales: reporte de um caso. Rev Asoc Odontol Argentina 89(5): 491-494.

5. Gómez VM (1997) Dientes natales y neonatales: revision de La literatura: primeira parte. Acta Odontol Venez 35(2): 54-60.

6. Zhu J, King D (1995) Natal and neonatal teeth. ASDC J Dent Chil 62(2): 123-128.

7. Leung AK, Robson WL (1986) Natal teeth. Am J Dis Child 140: 249-251.

8. Diniz MB, Coldebella CR, Zuanon AC, Cordeiro RC (2011) Oral abnormalities in preterm and low birth weight infants: the importance of the relationship between pediatricians and pediatric dentists. Revista Paulista de Pediatria 29(3): 449-455.

9. Villena RS, Corrêa MSNP (2009) Characteristics of the stomatognathic system in the newborn and more frequent anomalies. In: Corrêa MSNP (eds.), Pediatric dentistry 3 (edn.), Santos, Sao Paulo, Brazil, pp. 114-126.

10. Yared FNFG, Yared KFG (2002) Natal and neonatal teeth: diagnosis, treatment decisions and attention to early dental trauma. J Bras Odontopediatr Odontol Baby 5(25): 21-27.

11. Fierro MC, Bravo RL, Torres CF, Álvarez HC, Pérez FMA (2010) Natal Teeth, Case Report and Review. International Journal of Odontostomatology 4(2): 105-110.
12. Ulson RCB, Corrêa MSN, Lopes L D (2008) Neonatal tooth: case report. Rev Inst Ciênc Saúde 26(1): 130-134.

13. Soares Junior BA, Lábio RO, Albuquerque Júnior RLC, Barreto MAC, Gonçalves SRJ, et al. (2011) Traumatic lingual ulcer in a child (Riga-Fede disease): case report and literature review. Cad Grad Ciênc Bio Health 13(14): 137-142.

14. Moreira FCL, Gonçalves IMF (2010) Dentes natais e doença de Riga Fede. Rev Gaúcha Odontol 56(2): 257-261.

15. Cunha RF, Boer FAC, Torriani DD, Frossard WTG (2001) Natal and neonatal teeth: review of the literature. Pediatr Dent 23(2): 158-162.

16. Kates GA, Needdleman HL, Holmes LB (1984) Natal and neonatal teeth: a clinical study. J Am Dent Assoc 109(3): 441-443.

17. Leung AKC, Robson WLM (2006) Natal teeth: a review. J Natl Med Assoc 98(2): 226-228.

18. Corrêa MSNP, Villena RS, Frascino SMV (1998) Clinical evaluation of the characteristics of the oral cavity and the occurrence of abnormalities in newborns. ABO Nac Magazine 6(2): 96-102.

19. Sevalho ML, Hanan AS, Alves Filho AOA, Medina PO (2011) Natal teeth: clinical case report. ConScienta e Saúde 10(1): 160-165.
To Submit Your Article Click Here: Submit Article

DOI: $10.32474 /$ IPDOAJ.2020.04.000178

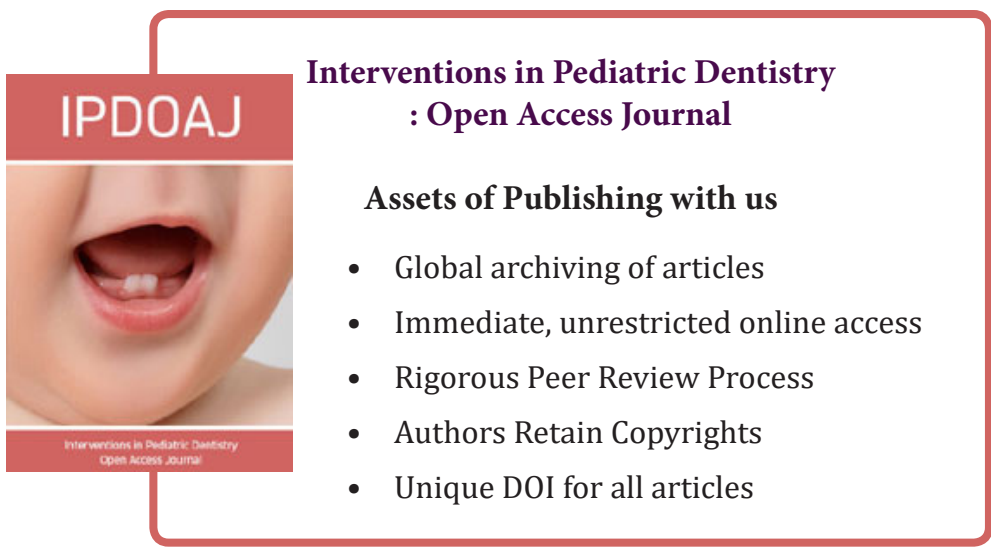

